\section{Editorial}

\section{Check for updates}

\section{OPEN ACCESS}

Received: Apr 28, 2020

Accepted: Apr 28, 2020

Correspondence to

Sean Kehoe

FIGO Gynaecological Cancer Committee,

Institute of Cancer \& Genomics, University

of Birmingham, Edgbaston, Birmingham B15

2TT, UK.

E-mail: sean.kehoe@spc.ox.ac.uk

Copyright (c) 2020. Asian Society of Gynecologic Oncology, Korean Society of

Gynecologic Oncology, and Japan Society of

Gynecologic Oncology

This is an Open Access article distributed

under the terms of the Creative Commons

Attribution Non-Commercial License (https://

creativecommons.org/licenses/by-nc/4.0/)

which permits unrestricted non-commercial

use, distribution, and reproduction in any

medium, provided the original work is properly

cited.

ORCID iDs

Sean Kehoe iD

https://orcid.org/0000-0003-3830-9532

Conflict of Interest

No potential conflict of interest relevant to this

article was reported.

\title{
FIGO staging in ovarian carcinoma and histological subtypes
}

\section{Sean Kehoe (D)}

FIGO Gynaecological Cancer Committee, Institute of Cancer \& Genomics, University of Birmingham, Birmingham, UK

- See the article "A retrospective study for investigating the relationship between old and new staging systems with prognosis in ovarian cancer using gynecologic cancer registry of Japan Society of Obstetrics and Gynecology (JSOG): disparity between serous carcinoma and clear cell carcinoma" in volume 31 , e45.

Over 70 years ago the concept of a structured staging process for gynecological cancers commenced-firstly with cervical cancer and with time expanding to all gynecological cancers. In the 1950's the responsibility of staging was devolved to International Federation of Gynecology and Obstetrics (FIGO) [1]. Since then, staging has been regularly reviewed and with the accumulation of data, accordingly revised to align staging to survival patterns. The main objective of staging is as an internationally agreed 'language', it also contributes to and facilitates other scientific endeavours, such as basic research, the collation of epidemiological data and registries. It also assists in directing therapeutic strategies for patients. Whilst staging remains the most important prognosticator for cancer outcomes, there are many other contributory variables, such as tumor mutational status and other molecular and serum tumor markers [2].

Ovarian carcinoma remains the most lethal of the gynecological cancers, with advanced disease (stage III/IV) constituting over $70 \%$ of cases at presentation and an associated 5-year survival at around $40 \%$. Ovarian cancer is a complex disease, with historically accepted hypotheses now been challenged. Most prominent, is the recognition that many, if not all, high grade serous carcinomas originate from the distal portion of the fallopian tubes [3]. This has implications regarding effective screening and the detection of disease at an early stage. Equally, the fallopian tube is the likely source for many primary peritoneal cancers. In FIGO staging fallopian tube, ovarian and peritoneal cancers are amalgamated into a single system [4].

Another advance is the elucidation of the carcinogenic developmental pathways in ovarian malignancies resulting in a stratification of the disease into 2 types: type I which contains mucinous, low grade serous, clear cell carcinomas, and type 2, the high grade serous, undifferentiated and carcinosarcomas [5]. Type 1 diseases are more indolent with a better survival pattern as compared to type 2 , which have greater aberrant mutational status and a more inherently aggressive tumor biology, albeit more chemo-sensitive.

A further change pertinent to the paper in the Journal relates to histological subtypes. Mucinous ovarian carcinomas once deemed relatively common are now considered rare. The actual origin of these tumors remains controversial [6]. However, the early cessation 
of a recent international trial on mucinous ovarian cancers, was due to the recognition on histopathological review, that many of the 'ovarian mucinous tumors' were in fact tumors of extra-ovarian origin [7]. It is now estimated that mucinous tumor account for $5 \%$ or less of ovarian cancer malignancies.

The paper by Yamagami et al. [8], analyzed a large Japanese ovarian cancer database to compare the stratification of women managed during 2004-2008 with staging revised to the 2014 FIGO criteria. The final cohort consisted of 9,749 women, and the 5-year overall survival was calculated within each stage, and where feasible within the sub-stages. The focus of the paper was on 2 groups: those with clear cell and mucinous carcinomas were combined as one $(\mathrm{C}+\mathrm{M})$, as were serous and endometrioid tumors $(\mathrm{S}+\mathrm{E})$. The premise for this was based on the known contrasting chemo-responsiveness of these groups. The preponderance of clear cell tumors within Japan's population, compared to Western countries, is also an important element in the study. Clear cell carcinomas constituted over $25 \%$ of the study population.

Reassuringly, the results confirmed a good correlation between outcomes and the 4 basic FIGO staging groups and many of the subgroups also performed well in distinguishing survival differences. Within stage 1 disease a statistically significant difference in outcome was noted between stage IA and ICI for the $\mathrm{C}+\mathrm{M}$ cohort, but not the $\mathrm{S}+\mathrm{E}$ group. Comparing stage IVA and IVB disease, again there was a significant difference in survival in the $\mathrm{C}+\mathrm{M}$ group, not found in the $\mathrm{S}+\mathrm{E}$ population. Caveats in these type of studies are inevitable, but are well highlighted by the authors, such as limited information on cytology of pleural effusions, and managing the challenging situation of palpably enlarged lymph nodes, without histological confirmation of disease. It is interesting to note that mucinous tumors contributed to $13.5 \%$ of the histological subtypes and it remains unknown how many of these cancers would today be categorized as 'non ovarian' in origin, and what impact that may have on the findings. However, the paper does highlight the unique scenario within Japanese and other Asian populations, and the impact of histology subgroupings on prognosis. The authors conclude that the sub-classification of stage IC may be too detailed.

This paper draws attention to certain elements of the staging process. Is staging sometimes too complex, and what other variables not included in staging influence prognosis? For example, there is an ever increasing number of molecular prognosticators and more sophisticated histopathological methods refining diagnoses. So where do these fit within the staging process? Obviously, not all countries will have the laboratory facilities to identify such markers. But by increasing the complexity of staging, endeavouring to encompass all known prognostic variables would render the system impractical, with the unintended consequence of reducing its accuracy and use in the global arena. Those directly involved in determining staging of cancers have debated these issues, concluding that maintaining the basic global 'language' remained paramount [9]. This, of course, is not an impediment to the development of more intricate parallel systems. These would likely necessitate the use of artificial intelligence to amalgamate clinical, demographic, laboratory and other relevant data to develop a more individualized prognosticator profile, permitting more succinct targeting of the most effective treatments for the specific tumor type and stage.

Data provided in this paper is valuable and useful evidence for ovarian cancer staging, but also emphasizes the ongoing need to develop novel therapies for those ovarian malignancies less responsive to present treatment strategies. 


\section{REFERENCES}

1. Odicino F, Pecorelli S, Zigliani L, Creasman WT. History of the FIGO cancer staging system. Int J Gynaecol Obstet 2008;101:205-10. PUBMED | CROSSREF

2. Lee YJ, Kim HS, Rim JH, Lee JY, Nam EJ, Kim SW, et al. Germline BRCA, chemotherapy response scores, and survival in the neoadjuvant treatment of ovarian cancer. BMC Cancer 2020;20:185. PUBMED | CROSSREF

3. Crum CP, McKeon FD, Xian W. The oviduct and ovarian cancer: causality, clinical implications, and "targeted prevention". Clin Obstet Gynecol 2012;55:24-35. PUBMED | CROSSREF

4. Berek JS, Kehoe ST, Kumar L, Friedlander M. Cancer of the ovary, fallopian tube, and peritoneum. Int J Gynaecol Obstet 2018;143 Suppl 2:59-78. PUBMED | CROSSREF

5. Terada KY, Ahn HJ, Kessel B. Differences in risk for type 1 and type 2 ovarian cancer in a large cancer screening trial. J Gynecol Oncol 2016;27:e25. PUBMED | CROSSREF

6. Cheasley D, Wakefield MJ, Ryland GL, Allan PE, Alsop K, Amarasinghe KC, et al. The molecular origin and taxonomy of mucinous ovarian carcinoma. Nat Commun 2019;10:3935. PUBMED | CROSSREF

7. Gore M, Hackshaw A, Brady WE, Penson RT, Zaino R, McCluggage WG, et al. An international, phase III randomized trial in patients with mucinous epithelial ovarian cancer (mEOC/GOG 0241) with long-term follow-up: and experience of conducting a clinical trial in a rare gynecological tumor. Gynecol Oncol 2019;153:541-8. PUBMED | CROSSREF

8. Yamagami W, Hagase S, Takahashi F, Ino K, Hachisuga T, Mikami M, et al. A retrospective study for investigating the relationship between old and new staging systems with prognosis in ovarian cancer using gynecologic cancer registry of Japan Society of Obstetrics and Gynecology (JSOG): disparity between serous carcinoma and clear cell carcinoma. J Gynecol Oncol 2020;31:e45. PUBMED | CROSSREF

9. O'Sullivan B, Brierley J, Byrd D, Bosman F, Kehoe S, Kossary C, et al. The TNM classification of malignant tumours-towards common understanding and reasonable expectations. Lancet Oncol 2017;18:849-51. PUBMED | CROSSREF 